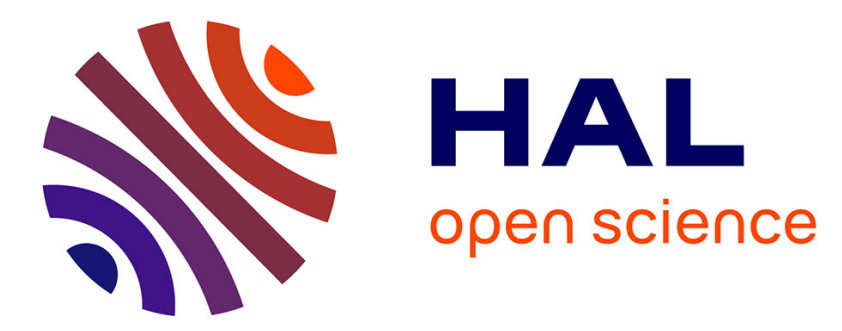

\title{
Electro-optic effects of aqueous fd-virus suspensions at very low ionic strength
}

H. Kramer, C. Graf, M. Hagenbüchle, C. Johner, C. Martin, P. Schwind, R. Weber

\section{- To cite this version:}

H. Kramer, C. Graf, M. Hagenbüchle, C. Johner, C. Martin, et al.. Electro-optic effects of aqueous fd-virus suspensions at very low ionic strength. Journal de Physique II, 1994, 4 (6), pp.1061-1074. 10.1051/jp2:1994184 . jpa-00248014

\section{HAL Id: jpa-00248014 https://hal.science/jpa-00248014}

Submitted on 1 Jan 1994

HAL is a multi-disciplinary open access archive for the deposit and dissemination of scientific research documents, whether they are published or not. The documents may come from teaching and research institutions in France or abroad, or from public or private research centers.
L'archive ouverte pluridisciplinaire HAL, est destinée au dépôt et à la diffusion de documents scientifiques de niveau recherche, publiés ou non, émanant des établissements d'enseignement et de recherche français ou étrangers, des laboratoires publics ou privés. 


\title{
Electro-optic effects of aqueous fd-virus suspensions at very low ionic strength
}

\author{
H. Kramer ( $\left.{ }^{1}\right)$, C. Graf ( $\left.{ }^{1}\right)$, M. Hagenbüchle ( $\left.{ }^{1}\right)$, C. Johner ( $\left.{ }^{1}\right)$, C. Martin ('), \\ P. Schwind $\left({ }^{2}\right)$ and R. Weber $\left({ }^{1}\right)$ \\ (') Fakultät für Physik, Universität Konstanz, 78434 Konstanz, Germany \\ ( $\left.{ }^{2}\right)$ Fakultàt fuir Bıologie, Universität Konstanz, 78434 Konstanz, Germany
}

(Received 5 November 1993, revsed 7 January 1994, accepted 18 February 1994)

\begin{abstract}
The orientation in external electric fields of rod-like fd-virus particles (length $\ell=895 \mathrm{~nm}$, diameter $d=9 \mathrm{~nm}$ ) in aqueous suspensions is examined by the electric birefringence method. In aqueous suspensions the negatively charged fd-particles are surrounded by a diffuse Debye cloud of counterions, which is characterized by the Debye-Hückel parameter $\kappa$. A special experimental set-up is used to vary the ionic strength of the suspension, i.e. the Debye-Hückel parameter, and therefore the electrostatic interparticle interaction. The birefringence signal $\Delta n$ is measured as a function of the strength and frequency of the applied electric field in suspensions of very low ionic strength $\left(10^{-6} \mathrm{M}-10^{-4} \mathrm{M}\right)$. At low field strengths Kerr-behaviour is found. From the dependence of the electric anisotropy $\Delta \alpha_{\mathrm{el}}$ on the Debye-Hückel parameter $\kappa$ it is concluded that the orientation of the fd-particles is correlated to an induced dipole due to a deformation of the diffuse Debye cloud. Saturation electric birefringence values are far from that theoretically expected. This can be interpreted as a destruction of the diffuse Debye cloud at high electric fields. At low field strengths the frequency dispersion below $1 \mathrm{kHz}$ of $\Delta n$ of the electrostatically interacting fd-virus suspensions shows anomalous behaviour. This negative electro-optic effect is an evidence for the orientation of the particle's long symmetry axis perpendicular to the applied electric field. The dispersion has a positive maximum at about $2 \mathrm{kHz}$. This maximum could be explaned by different frequency dependencies of the electric polarizabilities parallel and perpendicular to the long symmetry axis of the fd-rods.
\end{abstract}

\section{Introduction.}

Aqueous suspensions of charged macromolecules are very interesting two-component systems, built up by the charged macromolecular particles and the surrounding aqueous media. The particle charge leads to a substantial electrostatic interparticle interaction even at relatively small particle concentrations, when hard core interparticle interactions are still negligible. This allows the formation of ordered states of the macromolecules. These ordered states depend on the strength of electrostatic interaction. At weaker interaction a short-range order of the particles' centres of mass called liquid-like phase occurs. At stronger interaction a long-range order of the centres of mass called liquid-crystalline phase respectively for anisometric 
particles both a long range translational order and a long range orientational order can be found.

In this paper changes of the optical properties of aqueous fd-virus suspensions under the influence of an external electric field are examined by the electric birefringence method. The fd-virus is a filamenteous bacteriophage of length $\mathcal{l}=895 \mathrm{~nm}$, diameter $d=9 \mathrm{~nm}$, and very low polydispersity [1]. It has a rod-like shape and is optically anisotropic due to its molecular structure. On its surface approximately $10^{4}$ ionizible acid groups are located. In aqueous suspensions these give rise to a negative charge on each virus. With titration analysis a number of 8800 charged residues at the surface is found at $\mathrm{pH} 7.4$ [2]. These lead in highly deionized water to long-range electrostatic interactions, which can be screened by adding salt to the solution. The particle concentration of the fd-virus suspensions was fixed such that no or liquid-like order occurred. This means that in the absence of an external electric field the particles are isotropically oriented, the suspension is optically isotropic. Under the influence of an external field the fd-particles become oriented, the suspension is optically anisotropic and therefore birefringent. From the magnitude of the birefringence conclusions about the orientation of the fd-particles, the properties and the relevant orientation processes can be made. The field free relaxation after switching off the external electrical field contains information about the rotational diffusion of the particles [3].

Here we are interested in fd-virus suspensions at very low ionic strength. Under this condition the charged particles are surrounded by diffuse counterion clouds extending for several hundred nanometers. To our knowledge electro-optic measurements on charged particles with very extended counterion clouds have not yet been performed. We can show that the extended counterion clouds strongly contribute to the electro-optic properties of the charged particles. The extension of the counterion clouds determines the interparticle interaction and the interaction of the single particle with the external electric field, and therefore the orientation of the particle.

There are few theoretical publications which try to describe the influence of electric fields on diffuse counterion clouds but could not be tested yet because of the lack of experimental data. This theoretical work predicts quantitatively the polarizability of the counterion clouds $[4,5]$ and the destruction of the counterion clouds in high electric fields [6].

In the literature anomalous birefringence effects are described [7-10]. These phenomena are since over fifty years an object of controversial discussion which is mainly caused by insufficient characterization of the studied systems. The well characterized fd-virus particles in combination with our electro-optic methods allow measurements at very low ionic strengths and are therefore very appropriate to study these effects.

\section{Experimental section.}

MATERIALs. - A stock solution $(9 \mathrm{mg} / \mathrm{ml})$ of fd-virus particles was prepared with help of Professor Rasched (University of Konstanz) following a method of Marvin et al. [11]. Escherichia coli bacteria were infected with the fd-virus. After $8 \mathrm{~h}$ at $37^{\circ} \mathrm{C}$ the fd-virus bred rapidly by a factor of 1000 . The reproduction was stopped, and the virus was separated from the bacteria by several steps of precipitation and centrifugation. Finally the fd-virus suspension was ultracentrifuged in a $\mathrm{CsCl}$ gradient and dialyzed against a solution of $0.01 \mathrm{M} \mathrm{Tris} / \mathrm{HCl}$ to obtain a pure stock solution. The samples were prepared by diluting the stock solution with highly purified water $(R=18 \mathrm{M} \Omega)$. The actual concentrations of the samples were determined by their UV absorption at $\lambda=269 \mathrm{~nm}$ (extinction coefficient $\varepsilon=3.84 \mathrm{~cm}^{2} / \mathrm{mg}$ ), using a Beckmann spectrometer (DU-64, Darmstadt, Germany).

TUBE PUMP SYSTEM. - In a closed circuit, including the birefringence cell, the samples were deionized by pumping them with a tube pump through mixed-bed-ion-exchange resin (MB3, 
Serva Diagnostics, Heidelberg, Germany) until the desired conductivity was achieved [12]. The minimum ionic strength depends on the particle concentration. At this ionic strength the suspension could be considered free of small ions other than $\mathrm{H}^{+}$or $\mathrm{OH}^{-} \mathrm{NaCl}$ was added to the suspensions to obtain higher conductivities, i.e. smaller Debye clouds. All measurements were carried out at $20.0 \pm 0.2^{\circ} \mathrm{C}$. Conductivity measurements were performed using a Knick conductometer (Knick, Berlin, Germany).

ELECTRIC BIREFRINGENCE APPARATUS. - The electric birefringence apparatus is a commercial instrument (spectrometer DB 10, Suck, Siegen, Germany) and similar to that described elsewhere $[3,13,14]$. The birefringence was measured at the wavelength $\lambda=632.8 \mathrm{~nm}$. The effective voltage drop was measured at the cell terminals.

\section{Theory.}

The parameter describing the extension of the diffuse counterion cloud is the inverse DebyeHückel parameter, the Debye-Hückel length $\kappa^{-1}$, which is given by [15]

$$
\kappa^{-1}=\left(\frac{e_{0}^{2}}{\varepsilon_{\mathrm{r}} \varepsilon_{0} k_{\mathrm{B}} T} \sum_{i} c_{l} z_{1}^{2}\right)^{-1 / 2}
$$

where $z_{1}$ and $c_{1}$ are the charge number and the concentration of the charged species $i$. $\varepsilon_{0}$ is the permittivity of vacuum, $\varepsilon_{\mathrm{r}}$ the relative permittivity, $k_{\mathrm{B}}$ the Boltzmann constant, $e_{0}$ the elementary charge and $T$ the temperature. The ionic strength IS is defined by

$$
\mathrm{IS}=\frac{1}{2} \sum_{l} c_{\imath} z_{l}^{2}
$$

For small $\mathrm{NaCl}$ concentrations the ionic strength is proportional to the measured conductivity (IS $(\mu \mathrm{M}) \approx 9.3 \times$ conductivity $(\mu \mathrm{S} / \mathrm{cm}))[16]$ and the Debye-Hückel length $\kappa^{-1}$ can be calculated with good approximation by

$$
\kappa^{-1}=\frac{9.6(\mathrm{~nm})}{\sqrt{\mathrm{IS}}(\mathrm{mM})}
$$

The electric anisotropy $\Delta \alpha_{\mathrm{el}}$ of the fd-virus particles can be calculated from the Kerr-behaviour at small field strength by the Kerr-law

$$
\Delta n_{0}=\frac{\Delta n_{\mathrm{sat}} \Delta \alpha_{\mathrm{el}}}{15 k_{\mathrm{B}} T} E^{2}
$$

where $\Delta n_{0}$ is the measured birefringence, $E$ is the electric field strength, and $\Delta n_{\text {sat }}$ is the birefringence at infinite high field strength. Normally, when all particles are totally oriented in the field direction, from the saturation value $\Delta n_{\text {sat }}$ the optical anisotropy of the particles, which is determined by the molecular structure of the virus, can be calculated.

\section{Results and discussion.}

In a first part results obtained by transient electric birefringence are discussed. For aqueous fdvirus suspensions at small ionic strengths the steady-state birefringence signal $\Delta n_{0}$ was measured. Therefore the length of the electric field square pulses was chosen long enough to reach a stationary value of the signal. The pulse length in the range between 20 and $200 \mathrm{~ms}$ depended on the ionic strength. 
Figure 1 shows the field strength dependence of the birefringence signal $\Delta n_{0}$, normalized to the virus concentration, for various virus concentrations at minimal ionic strength $\left(10^{-6} \mathrm{M}\right)$. For small concentrations positive birefringence is measured, which increases with the electric field strength. At higher concentrations negative birefringence is found, slowly increasing with the electric field strength and changing at a larger field value, which depends on concentration, to positive birefringence. The concentrations in figure 1 are given in units of $c^{*}$, which is the overlap concentration of 1 particle/(length $\ell)^{3}$ i.e. $0.04 \mathrm{mg} / \mathrm{ml}$.

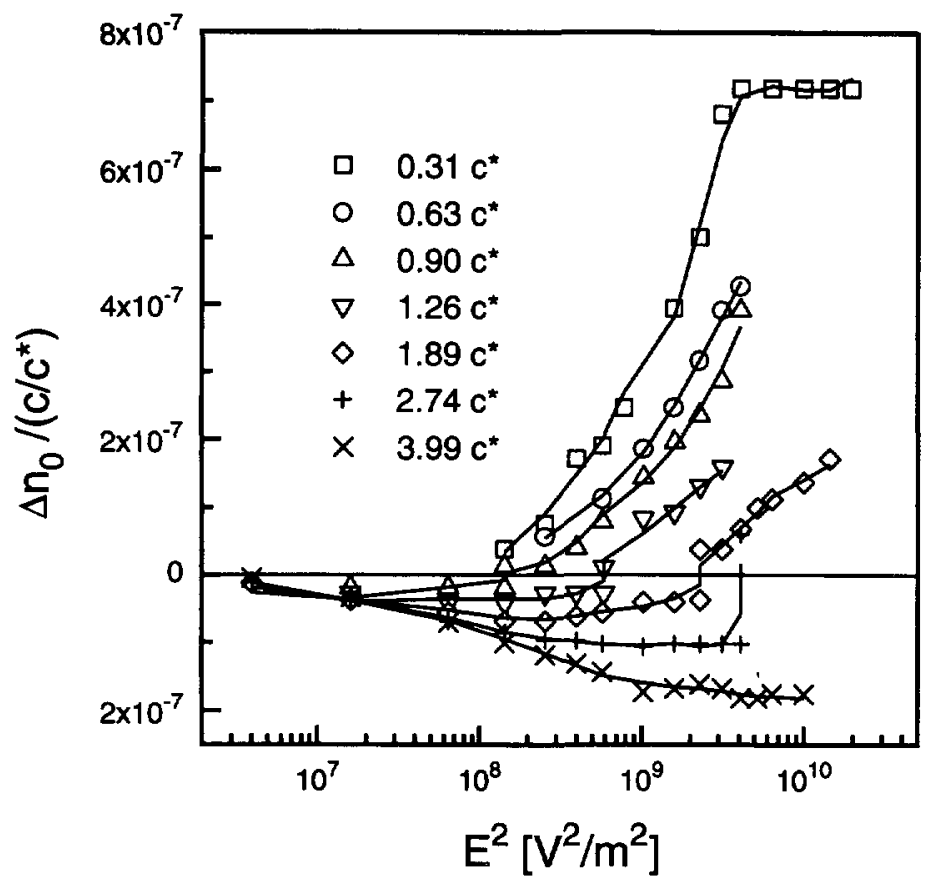

Fig. 1. - The specific birefringence of fd-virus suspensions at minimal conductivity at different fd-virus concentrations. They are normalized to the overlapping value $c^{*}$

The observed change of sign of the signal in this case from negative values at low field strengths and higher virus concentrations to positive values at higher field strengths has been observed in the literature for other rodlike particles but is not yet understood [3, 7-10, 17-19]. It is called anomalous birefringence and caused by the electrostatic interparticle interaction [3, $9,20]$.

To determine electro-optic parameters, as electric and optical anisotropy of the single fdvirus at minimal ionic strength, it is necessary to know the electric field range where the suspensions show Kerr-behaviour as a function of concentration. Figure 2 shows the signal $\Delta n_{0}$, normalized to the concentration and $E^{2}$, as a function of the virus concentration for different field strengths. This quantity is proportional to the Kerr-constant and Kerr-behaviour occurs when it is independent of the field strength. Figure 2 shows that this is the case at concentrations below $c^{*}$. Higher concentrations lead to anomalous birefringence and nonKerr-behaviour. Similar behaviour was found by Hoffmann, Krämer and Thurn for micelles and NaPSS $[9,10,21]$. One result is that the electric anisotropy can be determined from Kerrbehaviour only at particle concentrations below $0.5 c^{*}$ 


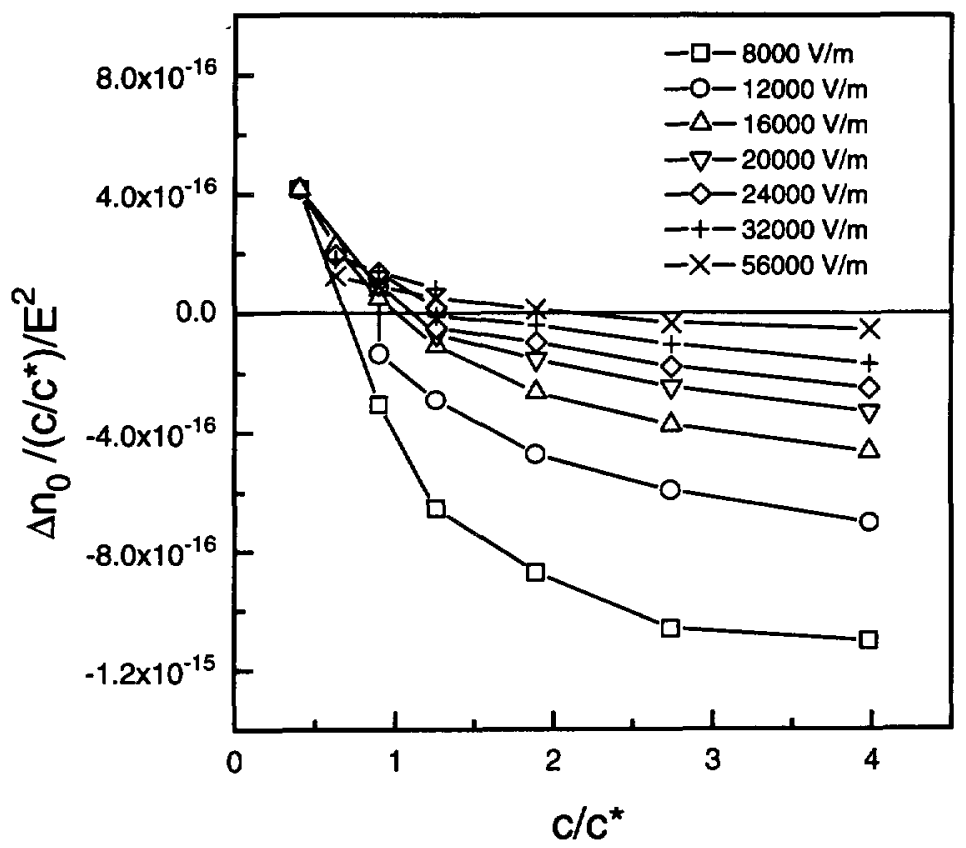

Fig. 2. - The specific birefringence of fd-virus suspensions normalized to the electric field strength at minimal conductivity for various electric field strengths.

One aim of this paper is to measure the dependence of the electric polarizability of the fdvirus particle on the Debye-Hückel length $\kappa^{-1}$ Therefore the field strength dependence of the transient electric birefringence signal $\Delta n_{0}$ was analyzed at a concentration well below $c^{*}$ [3]. Figure 3 shows $\Delta n_{0}$ under these conditions. A significant result of figure 3 is the strong dependence of the high field saturation value of $\Delta n_{0}$ on the different conductivities. For fdvirus suspensions Torbet and Maret [22] measured a saturation value of $\Delta n_{\text {sat }}\left(1 c^{*}\right)=$ $2.4 \times 10^{-6}$ at complete orientation of the particles in a magnetic birefringence experiment. This value is much larger than the values shown in figure 3 , where the highest values are about $30 \%$ of it. The saturation value is given by the optical anisotropy of the virus which is not influenced by the conductivity of the suspension at these low ionic strengths. For the optical anisotropy of fd-virus particles Torbet and Maret measured a value of $\Delta \alpha_{0}=2.44 \times$ $10^{-35} \mathrm{Fm}^{2}$. Also in electric field light scattering experiments [23] the measured value for the saturation is about four times smaller than the theoretically predicted value. Therefore we must conclude that the measured saturation values in figure 3 do not correspond with totally oriented particles. Kerr-behaviour is found at low electric field strengths, and with the help of the given saturation value of Torbet and Maret the electric anisotropy $\Delta \alpha_{\text {el }}$ is calculated with formulas (3) and (4) as a function of the Debye-Hückel length $\kappa^{-1}$

The values for $\Delta \alpha_{\text {el }}$ in figure 4 are about five orders of magnitude larger than the value of $\Delta \alpha_{0}$ given by Torbet and Maret. Therefore we must conclude that as long as the electric polarizability mechanisms leading to this high $\Delta \alpha_{\mathrm{el}}$-values are intact, the orientation of the particles is dominated by these mechanisms. Incomplete orientation and saturation effects at the moderate electric field strengths we are using in this experiments can be only explained by these electric polarizability mechanisms. However assuming that the particles stay intact at whatever high electric fields due to their optical anisotropy $\Delta \alpha_{0}$ the particles should orient completely in electric field direction. For the small $\Delta \alpha_{0}$-values of the fd-particles and for the electric field strengths we use in our experiments we are far away to reach this case. 


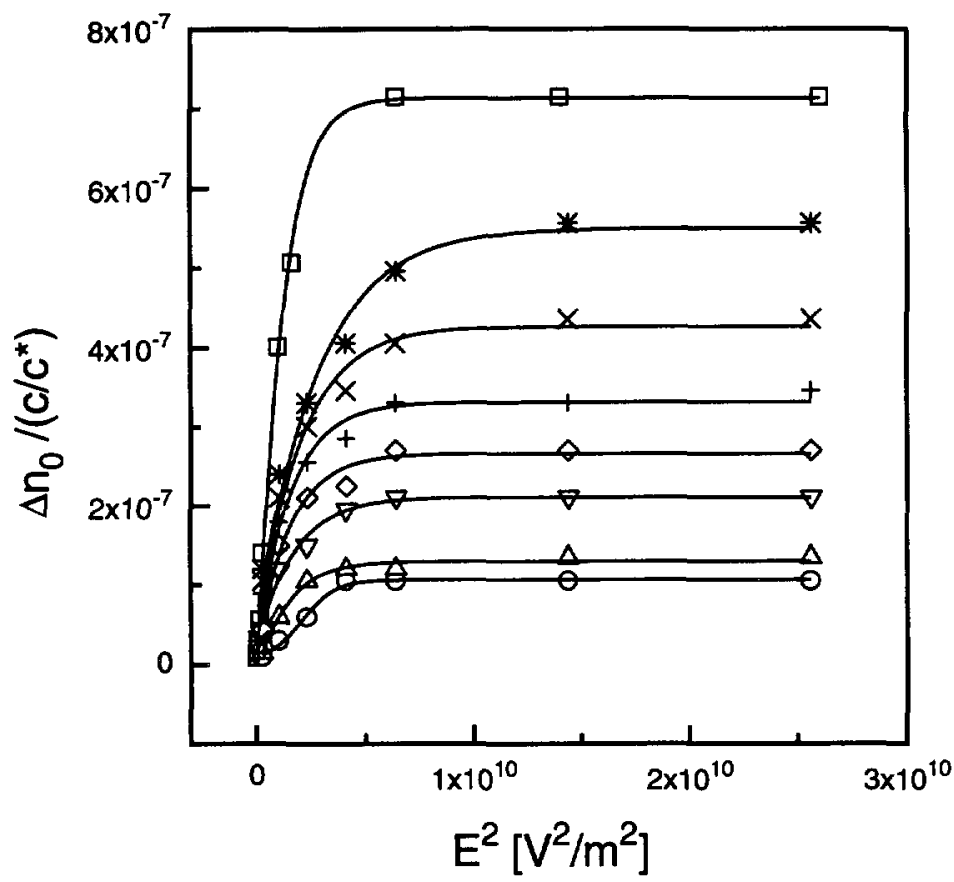

Fig. 3. - The birefringence of a fd-virus suspension with a concentration of $0.4 c^{*}$ for various conductivities. They are (starting from the top) : $0.22 \mu \mathrm{S} / \mathrm{cm} ; 1.3 \mu \mathrm{S} / \mathrm{cm} ; 1.61 \mu \mathrm{S} / \mathrm{cm} ; 1.97 \mu \mathrm{S} / \mathrm{cm}$; $2.4 \mu \mathrm{S} / \mathrm{cm} ; 3.15 \mu \mathrm{S} / \mathrm{cm} ; 5.2 \mu \mathrm{S} / \mathrm{cm} ; 6.7 \mu \mathrm{S} / \mathrm{cm}$.

Figure 4 shows a strong increase of $\Delta \alpha_{\text {el }}$ with $\kappa^{-1}$ of more than one order of magnitude in the $\kappa^{-1}$ range from 40 to $120 \mathrm{~nm}$. A similar linear behaviour was measured by Hogan et al. [24] and Rau and Bloomfield [25] on charged DNA rods at higher ionic strengths, and interpreted in the way that the electric anisotropy is dominated by the diffuse Debye cloud and not by counterions bound to or adsorbed on the macromolecular surface. Theoretical considerations of Rau and Charney [26] concerning DNA particles led them to the result that in the $\kappa^{-1}$ range below $10 \mathrm{~nm}$ adsorbed counterions are dominating the electric polarizability, at $\kappa^{-1}$-values above $20 \mathrm{~nm}$ the diffuse counterion cloud begins to dominate the electric polarizability. Rau and Charney could compare their results with experimental values up to $\kappa^{-1}=27 \mathrm{~nm}$ and give a nearly linear dependence of the electric polarizability parallel to the rod length axis $\alpha_{\mathrm{el}}^{\mathrm{pa}}$ on $\kappa^{-1}$ [27]. The results presented in this paper show the strong influence and the predominant effect of the diffuse counterion cloud on the electric polarization at higher Debye-Hückel lengths.

Fixman and Jagannathan $[5,28]$ showed that for rod-like particles at very low ionic strengths, when the Debye-Hückel length is in the range of the rod length, the magnitude of the electric polarizability $\alpha_{\mathrm{el}}^{\mathrm{pe}}$ perpendicular to the rod is comparable to the electric polarizability $\alpha_{\mathrm{el}}^{\mathrm{pa}}$ parallel to the rod and therefore contributes significantly to the electric anisotropy. Further, they showed that for extended counterion clouds the electrophoretic movement of the particle relative to the solvent causes an additional convective polarization which can dominate the electric polarization of the counterion cloud.

Our results yield that the fd-virus particles at low ionic strength are oriented in electric fields by an induced dipole mechanism caused by the polarization of their surrounding diffuse 


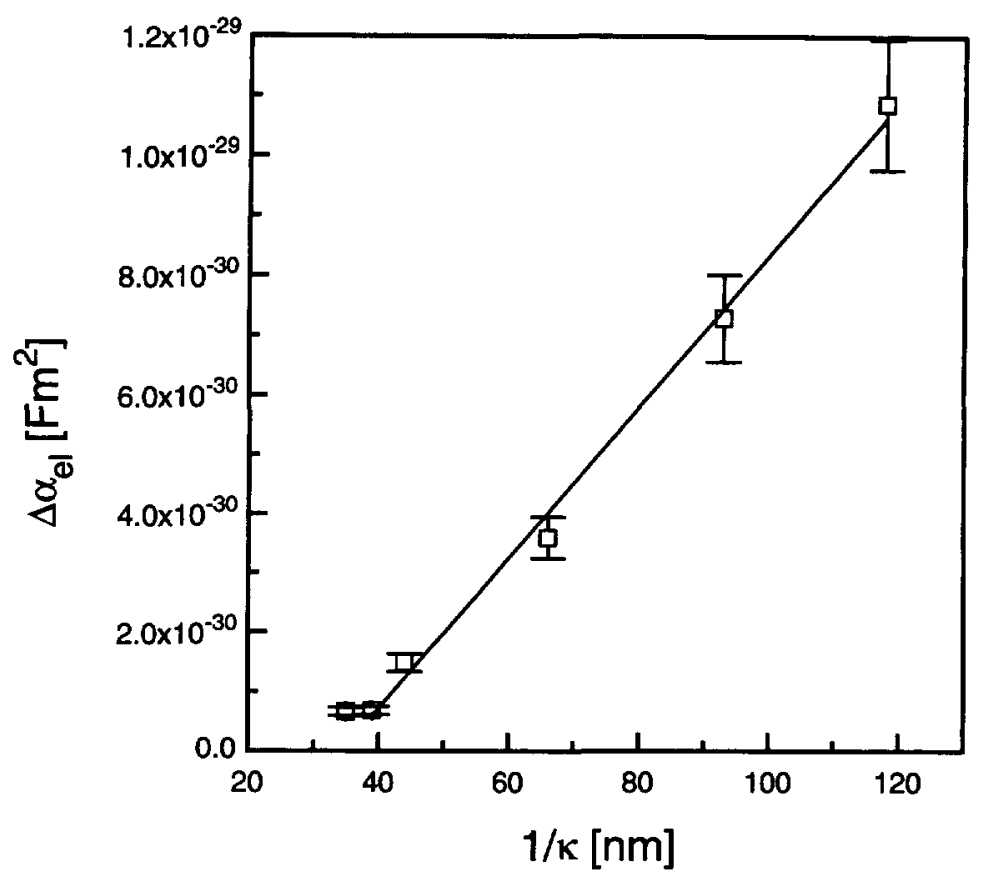

Fig. 4. - The electric anisotropy $\Delta \alpha_{\mathrm{el}}$ of the fd-virus in aqueous suspension.

counterion clouds. This orientation mechanism explains why the particles cannot be fully oriented at higher electric field strengths. In the literature several authors discuss theoretically the influence of high electric fields on the diffuse ion cloud [6, 29-31]. Considerations of Fixman and Jagannathan on spherical polyelectrolytes show that at moderate electric field strengths of several hundred $\mathrm{V} / \mathrm{cm}$ the induced dipole is no longer proportional to the electric field, and the ion cloud starts to be stripped away. Yoshida et al. showed by Monte Carlo simulations similar behaviour for rod-like particles. A destruction of the diffuse ion cloud leads to a destruction of the orientation mechanism, and therefore to saturation effects and incomplete orientation for rod-like particles at higher electric fields. Figure 3 shows for the virus-suspensions at different ionic strengths a common start of saturation effects at an electric field strength of about $10^{5} \mathrm{~V} / \mathrm{m}$. An interpretation of this effect could be that at this field strength the stripping effect starts and the measured saturation value of the birefringence is mainly given by the ionic strength dependent electric anisotropy of the particles. This would lead to decreasing the observed saturation values with increasing ionic strength.

In figure 1 for fd-virus suspensions at minimal ionic strength, low electric field strengths and particle concentration above $0.5 c^{*}$ negative birefringence signals are found. This effect is called the low field anomaly of the electric birefringence. From recent studies it is known that the low field anomaly is a collective effect of electrostatically interacting rods in the suspension which prefer to orient perpendicular to the applied electric field [3, 9, 23]. Figure 5 shows a pure anomalous signal which is not superimposed by normal birefringence. This implies that at sufficiently strong electrostatic interaction the orientation mechanism leading to anomalous orientation is not superimposed by normal orientation mechanisms. To our knowledge such purely anomalous signals were not yet measured. Low field anomaly occurs in suspensions under conditions where liquid-like structure is found in static light scattering experiments [32]. Higher electric fields destroy the low field anomaly. Figure 6 shows the 


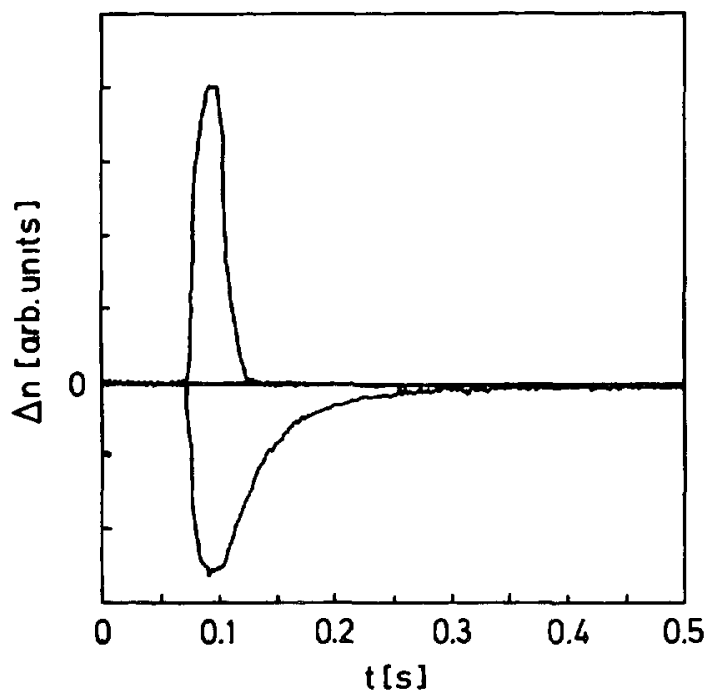

Fig.5.

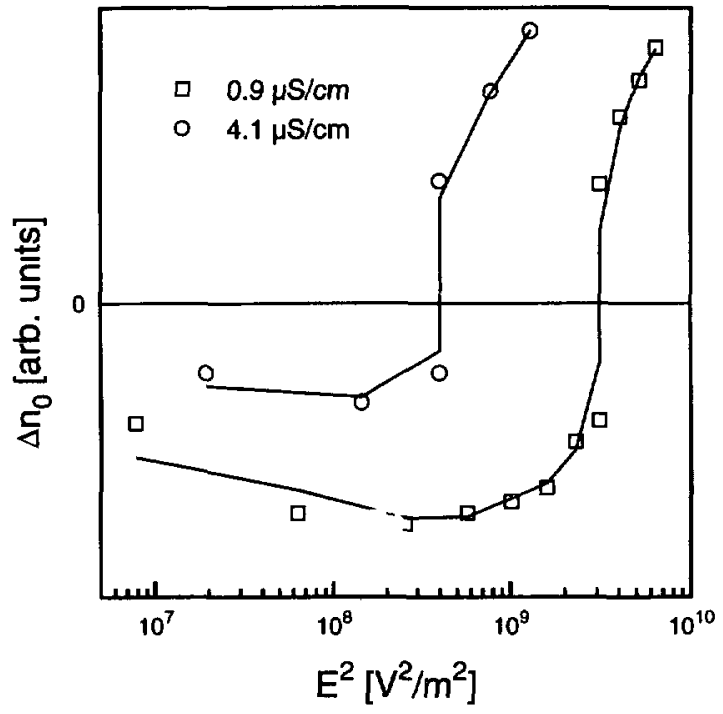

Fig. 6 .

Fig. 5. - The birefringence signal of a fd-virus suspension with concentration $1 c^{*}$. Positive signal : conductivity $1 \mu \mathrm{S} / \mathrm{cm}$, electric field strength $160 \mathrm{~V} / \mathrm{cm}$; Negative signal : conductivity $0.45 \mu \mathrm{S} / \mathrm{cm}$, electric field strength $60 \mathrm{~V} / \mathrm{cm}$.

Fig. 6. - The birefringence of a fd-virus suspension with concentration $2.1 c^{*}$ for two conductivities.

anomalous birefringence for a fd-virus suspension at two different conductivities. It is well demonstrated that both screening of the electrostatic interaction and high electric fields hinder anomalous orientation. An analogous behaviour was found by Asai and Watanabe in TMVsuspensions [8]. Figure 5 shows birefringence signals at two different conductivities and electric field strengths.

The order of magnitude of the anomalous effect can be calculated from the results in figure 1 . In figure 1 the highest normalized negative value reaches about $30 \%$ of the highest positive value, which is about $30 \%$ of the value given by Torbet and Maret for complete orientation [22]. Therefore the maximum negative value reaches about $9 \%$ of the value for complete orientation in electric field direction. At complete orientation of the rods perpendicular to the electric field this value should reach $50 \%$.

In the last few years different ideas to understand the low field anomaly were presented [3, 9, 20]. The suggestions of Hoffmann et al. and Cates were developed with the aid of results obtained for micellar and NaPSS-suspensions. Neither micelles nor NaPSS are ideal rods. Therefore in experiments on these systems effects were found which are specific for these systems, but are not found in our nearly ideal systems of fd-virus suspensions at very low ionic strengths and therefore may not constitute a general model for the low field anomaly. However two basic points enter all suggestions explaining the low field anomaly. The first point is that the low field anomaly is a collective effect of interacting rodlike particles. The second point is that the low field anomaly is the result of a preferable particle orientation perpendicular to the external electric field direction, which can be only explained with a not negligible electric polarization perpendicular to the rod length axis. Both points are fulfilled for our particles with extended Debye clouds. 
Measurements of Hoffmann et al. [9, 10,33] show a disappearance of the anomaly at higher particle concentrations which is not found for our systems up to a phase-transition to a liquidcrystalline phase at about $10 c^{*}$. The model of Hoffmann et al. of a hindered longitudinal polarization in a certain concentration range cannot explain pure anomalous birefringence signals as they are measured for the fd-virus suspensions.

Another idea seems to be more successful. A system of electrostatically interacting polarizable rods will orient in an electric field in a way to minimize the free energy of the system. Odijk showed [34] that the free energy as a function of the particles positions and orientations is the sum of an unimportant constant, an orientational entropy term, a part describing the interparticle interaction and a part describing the interaction of the single rod with the external electric field. In our case, where the particles are strongly electrostatically interacting at small external electric fields the free energy should be dominated by the part describing the interparticle interactions. At high electric fields the interaction of the single rod with the electric field dominates the free energy [20]. Therefore at high electric fields the particles orient in field direction because of geometric reasons the polarizability along the rod axis is higher than the polarizability perpendicular to the rod axis.

To construct a model for the low field anomaly the interparticle interaction should favour a perpendicular orientation of the rods with respect to the electric field at small electric field strengths. Cates [20] proposed an interparticle interaction which leads to pre-smectic clusters of the rodlike particles. For energetical reasons in these clusters the rods should be oriented perpendicular to the electric field. Those clusters are not found in our systems and they are very unlikely because the phase transition at $10 c^{*}$ leads to a columnar and not a smectic phase. Further, purely anomalous birefringence signals are found for fd-virus suspensions down to $0.5 c^{*}$ and for TMV-suspensions down to $0.05 c^{*}[14]$. In the model of Cates this would imply that down to these low concentrations all particles should be organized in clusters. Single rods showing normal orientation and destroying the purely anomalous signals should not occur. This behaviour would be in contradiction to experimental and theoretical knowledge.

The interparticle interaction known from two electrostatically interacting rods seems to explain better the low field anomaly. Two repulsive electrostatically interacting rods want, due to the electrostatic torque, to twist away from a parallel configuration in order to minimize the repulsive force. Supposing that this behaviour is still valid in a multi-rod system, this interaction would hinder the rods to orient in the external electric field direction because this would imply a parallel orientation between the particles. At strong interparticle interaction an orientation of the rods with tendency perpendicular to the external field could be energetically favoured, because this still allows multiple orientation possibilities of the rods. However we admit that our results need additional theoretical work to be fully understood.

In the remaining part of this paper measurements of fd-virus suspensions in alternating electric fields are discussed. In the case of a sinusoidal field the frequency dependent electric birefringence signal $\Delta n(\omega, t)$ is given by

$$
\Delta n(\omega, t)=\Delta n_{\mathrm{sta}}(\omega)+\Delta n_{\mathrm{alt}}(\omega) \cos (2 \omega t-\varphi)
$$

where $\Delta n_{\text {sta }}(\omega)$ is a stationary time independent component, $\Delta n_{\text {alt }}(\omega)$ is the amplitude of the alternating component and $\varphi$ is the phase shift between the alternating component and the sinusoidal electric field. In the birefringence experiment the quantity $\Delta n_{\max }(\omega):=\Delta n_{\text {sta }}(\omega)+$ $\Delta n_{\text {alt }}(\omega)$ is measured.

The figures 7 to 11 show $\Delta n_{\max }$ as a function of the frequency $f=\omega / 2 \pi$ of the electric field for various fd-virus concentrations and electric field amplitudes at minimum ionic strength. At low frequencies of about $10 \mathrm{~Hz}$ the behaviour known from literature is found [3]. Suspensions of smaller fd-virus concentration show normal behaviour that means a positive signal, 


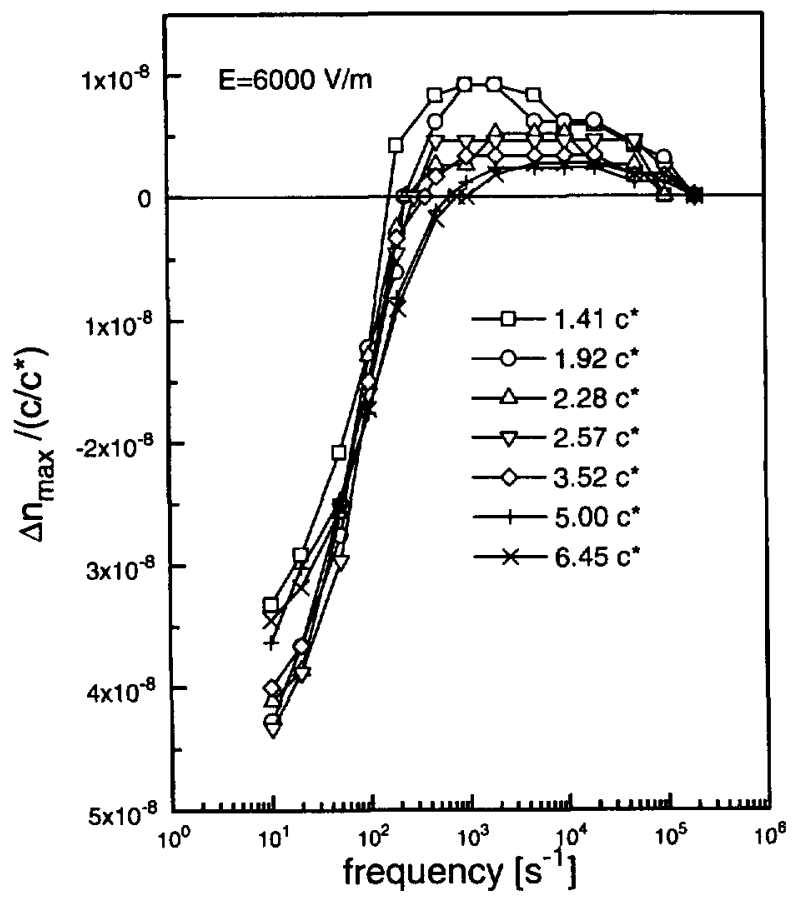

Fig. 7. - The specific birefringence of fd-virus suspensions at minimal conductivities and an amplitude of the sinusoidal electric field of $6000 \mathrm{~V} / \mathrm{m}$.

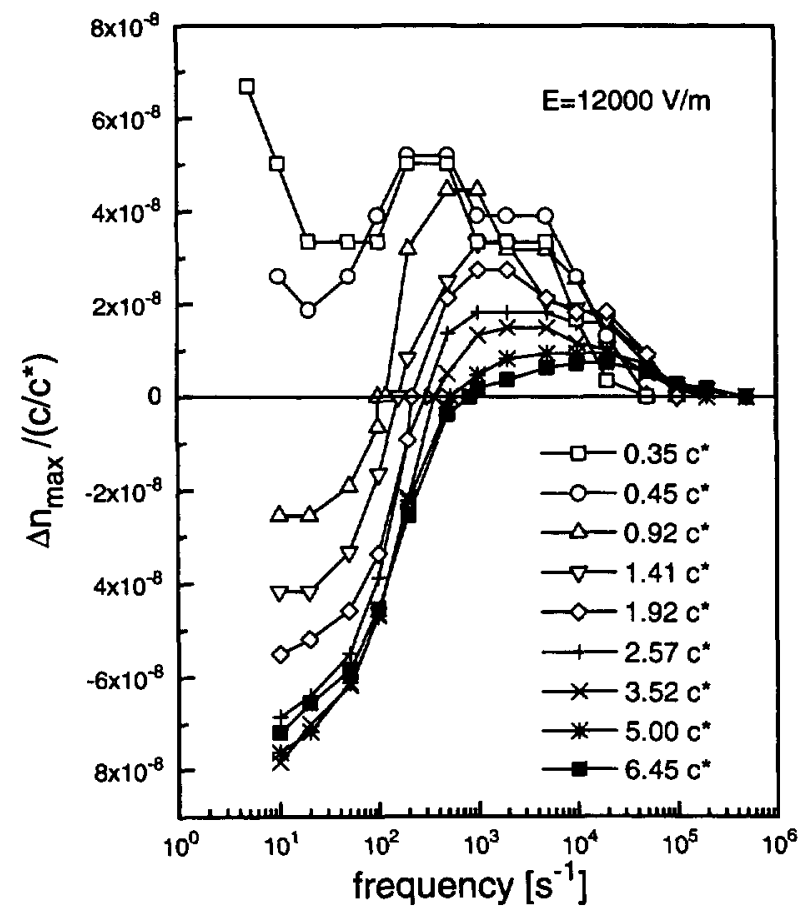

Fig. 8. - The specific birefringence of fd-virus suspensions at minimal conductivities and an amplitude of the sinusoidal electric field of $12000 \mathrm{~V} / \mathrm{m}$. 


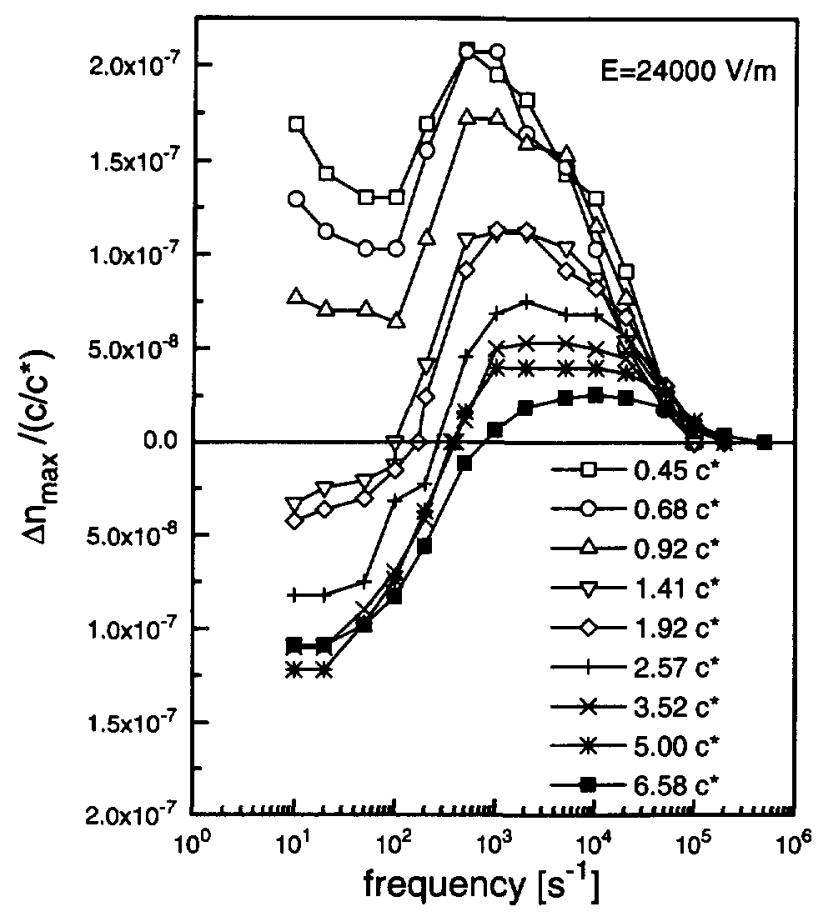

Fig. 9. - The specific birefringence of fd-virus suspensions at minimal conductivities and an amplitude of the sinusoidal electric field of $24000 \mathrm{~V} / \mathrm{m}$.

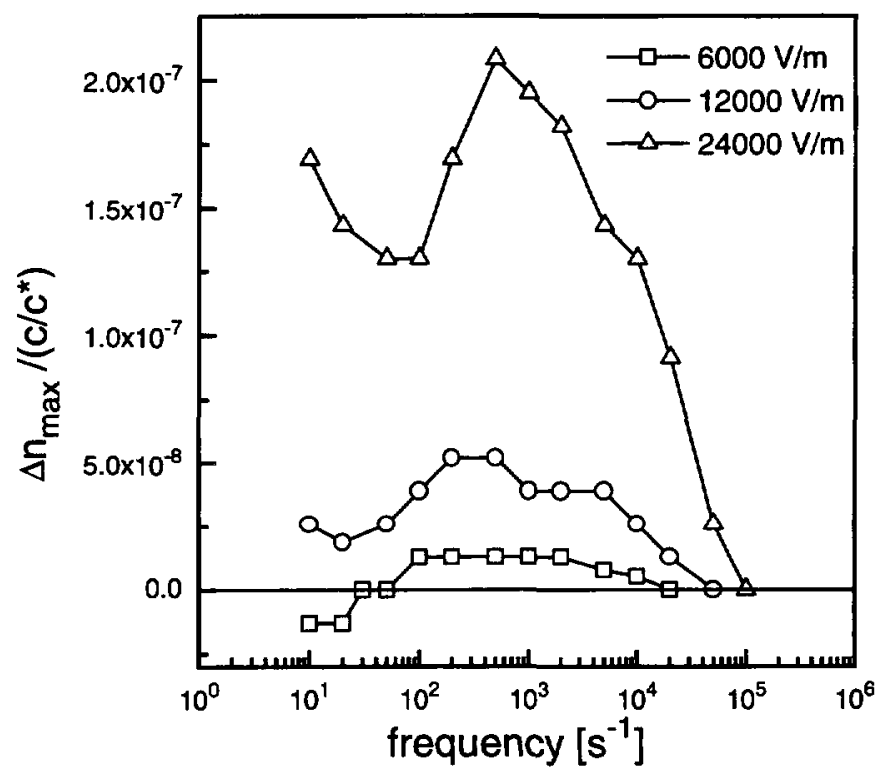

Fig. 10. - The specific birefringence of a fd-virus suspensions with concentration $0.5 c^{*}$ at minimal conductivity and various amplitudes of the sinusoidal electric field. 


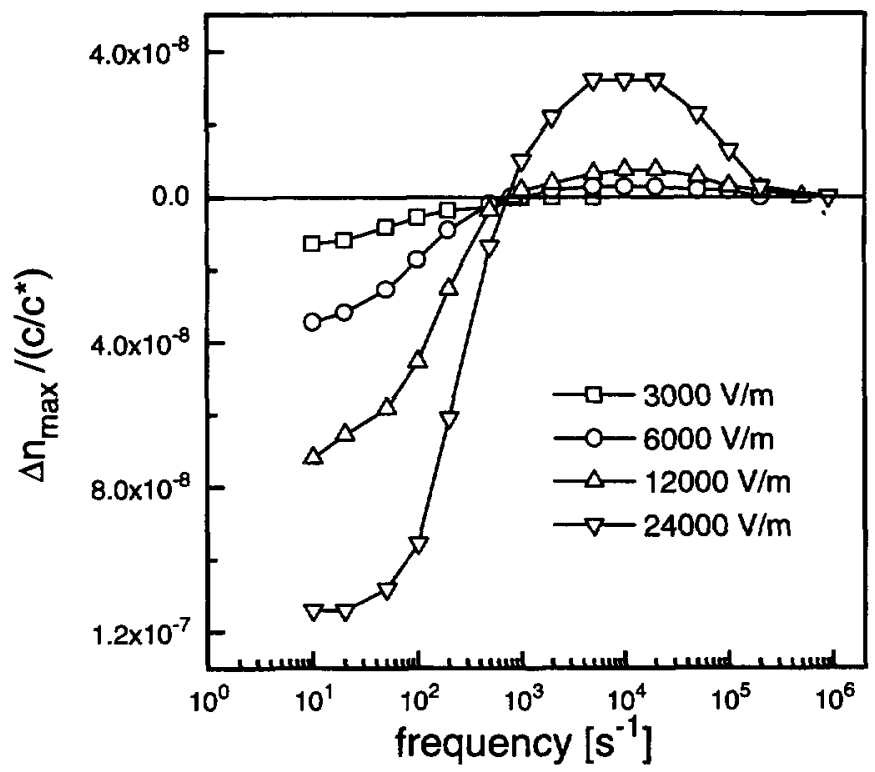

Fig. 11. - The specific birefringence of a fd-virus suspensions with concentration $6.45 c^{*}$ at minımal conductivity and various amplitudes of the sinusoidal electric field.

suspensions of concentration above the overlap concentration show anomalous, negative behaviour. Suspensions showing anomalous behaviour at low frequencies lose this with increasing frequency and with increasing electric field amplitude.

For all suspensions a positive maximum of the signal is found at frequencies of about $2 \mathrm{kHz}$. The disappearance of negative signals and the positive maximum of the signal at about $2 \mathrm{kHz}$ could be explained by the frequency dependencies of the electric polarizabilities parallel and perpendicular to the rod length axis. The measured signal at small electric field strength is proportional to the electric anisotropy $\Delta \alpha_{\text {el }}$ (see Eq. (4)), which is the difference of the polarizabilities parallel and perpendicular to the rod length axis.

$$
\Delta \alpha_{\mathrm{el}}=\alpha_{\mathrm{el}}^{\mathrm{pa}}-\alpha_{\mathrm{el}}^{\mathrm{pe}}
$$

A maximum of the signal with frequency at constant optical anisotropy and at constant electric field amplitude can be only explained with a maximum in the dispersion characteristic of the electric anisotropy $\Delta \alpha_{\mathrm{el}}$. In the case of a significant perpendicular polarizability, this can be caused by different dispersion behaviours of the polarizabilities parallel and perpendicular to the rod. Fixman and Jagannathan showed that the perpendicular polarizability takes a considerable magnitude in the case of extended diffuse counterion clouds [5]. Additionally the existence of anomalous birefringence is not explainable without such a significant polarizability $[9,14,20]$. A maximum in the dispersion of the electric anisotropy $\Delta \alpha_{\text {el }}$ occurs when the perpendicular polarizability decreases faster with the frequency than the parallel polarizability. The strong decrease of the perpendicular polarizability leads also to the disappearance of the anomalous birefringence.

One suggestion to understand the frequency dependent behaviour of the parallel and perpendicular polarizabilities is the following. A shift of the counterion cloud perpendicular to the rod length axis causes that large parts of the cloud come closer to the rod in regions of a 
stronger rod potential. This means that the counterions are stronger bound and that their translation diffusion constant is lower. Shifts of the counterion cloud parallel to the rod length axis cause that large parts of the cloud move at constant distance of the rod on equipotential surfaces. This fact might cause, that with increasing frequency the perpendicular polarization can harder follow the stimulating electric field than the parallel polarization. Therefore the two components of the polarizability show different behaviour with increasing frequency dispersion.

At frequencies in the range between $10^{5}$ and $3 \times 10^{5} \mathrm{~Hz}$ the birefringence signal vanishes. The vanishing of the signal and therefore the impossibility to orient the fd-particles at higher frequencies can be easily understood. At minimum ionic strength the diffuse counterion cloud surrounding the particle is mainly built up by $\mathrm{H}^{+}$counterions originating from the acid groups located on the virus surface. The orientation of the particle is therefore due to the polarization of the $\mathrm{H}^{+}$counterion cloud that means a shifting of the cloud relative to the negatively charged rod. The relaxation time and the reorientation time of such an induced dipole can be approximately calculated by a formula given by Oosawa $[35,36]$ :

$$
\tau=\frac{\ell^{2}}{(2 \pi)^{2} D}
$$

where $\mathcal{P}$ is the rod length, and $D$ the translation diffusion constant of the counterions. Using for $D$ the value for free $\mathrm{H}^{+}$ions at $20^{\circ} \mathrm{C}$ of about $8.5 \times 10^{-9} \mathrm{~m}^{2} / \mathrm{s}$ [37], which is possible because the ions are very weakly bound, leads to a time $\tau$ of about $2.4 \times 10^{-6} \mathrm{~s}$. This corresponds to a frequency of about $400 \mathrm{kHz}$, which is very close to the frequency at which the signals vanish i.e. the induced dipole can no longer follow the stimulating electric field and the rod is no longer oriented.

\section{Conclusions.}

The orientation behaviour of rod-like fd-virus particles in aqueous suspensions of very low ionic strength has been examined. It was demonstrated that at very low ionic strength $\left(10^{-5} \mathrm{M}-10^{-6} \mathrm{M}\right)$ the particles are oriented by an induced dipole mechanism due to the anisotropic polarization of their diffuse Debye cloud. This is in good agreement with predictions by Rau et al. [4] and Fixman et al. [5]. It is shown, that the counterion cloud is stripped away from the negatively charged rod at electric field strengths of about $10^{5} \mathrm{~V} / \mathrm{m}$. From our measurements we conclude that the rods possess a significant perpendicular polarizability, which leads to anomalous particle orientation in loqw electric fields in the case of electrostatically interacting rods. Models describing this anomalous orientation are discussed, but need additional theoretical work to be fully understood. In alternating electric fields the different dispersion behaviour of the polarizabilities parallel and perpendicular to the rod length axis could lead to a maximum of the measured signals at about $2 \mathrm{kHz}$ and to the disappearance of anomalous orientation at frequencies above $10^{2} \mathrm{~Hz}$. For alternating electric fields above $3 \times 10^{5} \mathrm{~Hz}$ no orientation of the particles is possible, which can be explained by the polarization of the diffuse counterion cloud which is limited by the diffusion of the $\mathrm{H}^{+}$counterions.

\section{Acknowledgments.}

This work was supported by the Deutsche Forschungsgemeinschaft (SFB 306). 


\section{References}

[1] Newman J., Swinney H., Day L. A., J. Mol. Bılol. 116 (1977) 593.

[2] Zimmermann K., Hagedorn H., Heuck C. C., Hinrichsen M., Ludwig H., J. Bıol. Chem. 261 (1986) 1653.

[3] Kramer H., Deggelmann M., Graf C., Hagenbüchle M., Johner C., Weber R., Macromolecules 25 (1992) 4325.

[4] Rau D. C., Charney E., Biophys. Chem. 14 (1981) 1.

[5] Fixman M., Jagannathan S., J. Chem. Phys. 75 (1981) 4048.

[6] Yoshida M., Kikuchi K., Maekawa T., Watanabe H., J. Phys. Chem. 96 (1992) 2365.

[7] Lauffer M. A., J. Am. Chem. Soc. 61 (1939) 2412.

[8] Asai H.. Watanabe N.. Biopolymers 15 (1976) 283.

[9] Hoffmann H., Krämer U., Thurn H., J. Phys. Chem. 94 (1989) 2027.

[10] Krämer U., Hoffmann H., Macromolecules 24 (1991) 256.

[11] Marvin D. A., Wachtel E. J., Nature 253 (1975) 19.

[12] Deggelmann M., Palberg T., Hagenbüchle M., Maier E. E., Krause R., Graf C., Weber R., J. Col. Int. Sci. 143 (1991) 318.

[13] Fredericq E., Houssier C., Electric Dichroism and Electric Birefringence (Clarendon Press, Oxford, 1973).

[14] Kramer H., PhD Thesis, Hartung-Gorre Verlag, Konstanz (1993).

[15] Hunter R. J., Zeta Potential in Colloid Science Principles and Applications (Academic Press, London, 1981).

[16] Gerthsen, Kneser, Vogel, Physik (Springer-Verlag, Berlin, 1982).

[17] Mueller H., Sakmann B. W., Phys. Rev. 56 (1939) 615.

[18] O'Konski C. T., Zimm B. H., Science 111 (1950) 113.

[19] Oppermann W., Makromol. Chem. 189 (1988) 2125.

[20] Cates M. E., J. Phys. II France 2 (1992) 1109.

[21] Thurn H., Löbl M., Hoffmann H., J. Phys. Chem. 89 (1985) 517.

[22] Torbet J., Maret G., Biopolymers 20 (1981) 2657.

[23] Kramer H., Martin C., Graf C., Hagenbuiche M., Johner C., Weber R., submitted to Prog. Coll. Int. Sci.

[24] Hogan M., Dattagupta N., Crothers D. M., Biochemistry 75 (1978) 195.

[25] Rau D. C., Bloomfield V. A., Biopolymers 18 (1979) 2783.

[26] Rau D. C., Charney E., Biophys. Chem. 17 (1983) 35.

[27] Rau D. C., Charney E., Biophys. Chem. 14 (1981) 1.

[28] Fixman M., J. Chem. Phys. 75 (1981) 4040.

[29] Fixman M., Jagannathan S., Macromolecules 16 (1983) 685.

[30] Rau D. C., Charney E., Macromolecules 16 (1983) 1653.

[31] Altig J. A., Wesenberg G. E., Vaughan W. E., Biophys. Chem. 24 (1986) 221.

[32] Graf C., Deggelmann M., Hagenbüchle M.. Kramer H., Krause R., Martin C., Weber R., $J$. Chem. Phys. 95 (1991) 6284.

[33] Krämer U., PhD Thesis, University of Bayreuth (1990).

[34] Odijk T., Laser Light Scattering in Biochemistry, S. E. Harding. D. B. Sattele, V. A. Bloomfield Eds., Roy. Soc Chem., London (1992).

[35] Oosawa F., Biopolymers 9 (1970) 677.

[36] Stoylov S. P., Electro-Optics Theory, Techniques, Applications (Academic Press, London, 1991).

[37] American Institute of Physics Handbook (McGraw-Hill Book Comp., New York, 1972). 